\title{
Hoja de ruta para avanzar hacia una administración pública moderna en Cuba
}

\section{Roadmap to advance to a modern public administration in Cuba}

\author{
Mariluz Llanes Font ${ }^{1}$ \\ Yudith Salvador Hernández ${ }^{2}$ \\ Elizabeth Aguilera Díaz ${ }^{3}$ \\ Pedro Escalona Ávila 4
}

Fecha de recepción: 14 de agosto del 2018

Fecha de aceptación: 19 de diciembre de 2018

\begin{abstract}
Resumen
Las personas autoras proponen como objetivo general analizar un conjunto de criterios que propicien el establecimiento del gobierno electrónico en Cuba, a partir de un instrumento que instituya el ámbito de planificación, ejecución y mejoras del gobierno electrónico para los diferentes actores involucrados. Se analizan los argumentos implícitos en los documentos del Partido Comunista y la Agenda 2030 del Desarrollo Sostenible que sustentan el establecimiento del gobierno electrónico. Se esboza de este sus características esenciales, el punto de partida en el contexto local y se describen los pasos que permitirán la implementación de forma coherente del gobierno electrónico para reducir la complejidad de la burocracia estatal, simplificar y mejorar la interacción entre la ciudadanía y los diferentes organismos públicos y avanzar hacia una administración pública más transparente, participativa, democrática y equitativa. En sus conclusiones se destaca que la finalidad esencial del gobierno electrónico es incrementar la calidad de los servicios y productos públicos que la administración pública suministre a la ciudadanía. Es un proceso complejo que requiere de tiempo y no se trata solo de que la ciudadanía pueda estar informados del quehacer de la administración pública y de pagar los impuestos por internet, sino que además puedan tener una discusión pública acerca de cómo se gastan esos impuestos. El desafío es ir más allá de los modelos unidireccionales de servicio y políticas públicas, se trata de la inclusión del ciudadano dentro de la toma de decisiones durante todo el ciclo de vida de la gestión pública.

Palabras clave: Gobierno electrónico; Gobierno digital; Gestión pública; Interacción ciudadanogobierno; Contexto local cubano.
\end{abstract}

1. Universidad de Holguín, Cuba, Profesora-Investigadora, mlfon@uho.edu.cu; mllfont62@gmail.com

2. Universidad de Holguín, Cuba, Profesora-Investigadora, ysalvadorh@uho.edu.cu

3. Universidad de Holguín, Cuba, Profesora-Investigadora, elizabethad@uho.edu.cu

4. Universidad de Holguín, Cuba, Profesor-Investigador, pedroe@uho.edu.cu

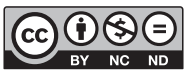




\begin{abstract}
The authors propose the analysis a set of criteria to favor the establishment of the electronic government in Cuba as the general objective, starting from an instrument that institutes the planning, executing and improving scope of the electronic government for the actors involved. The implicit arguments are analyzed in documents belonging to the Communist Party and the agenda 2030 on sustainable development that back up the establishment of the electronic government. The essential characteristics are outlined, the point of departure in the local context and the steps that allow the implementation of the electronic government in a coherent form are described, in order to reduce the complexity of the estate bureaucracy, to simplify and improve the interaction between the people and the different public administrations and advance towards a public administration more transparent, participative, democratic and with equality. In its conclusions it highlights that the essential purpose of the electronic government is increasing the quality of the services and public products, which the public administration supply to the people.

KeyWords: Electronic government; Digital government; Management public; Interaction citizengovernment; Cuban local context.
\end{abstract}

\title{
Introducción
}

Las nuevas formas de interacción entre la ciudadanía y el Estado a partir del uso de las tecnologías de la información y comunicación (TIC) para el mejoramiento de la gestión pública ya no se cuestionan. A través de ellas, las administraciones buscan reducir la complejidad propia de las burocracias estatales, simplificar y agilizar la interacción entre el ciudadano y los diferentes organismos públicos y avanzar hacia una administración sin papeles. Este tipo de gestión se ha denominado gobierno electrónico o gobierno digital. Es suficiente referirse a una definición de gobierno electrónico, de manera que resulta útil adoptar la de la Carta Iberoamericana de Gobierno Electrónico, explicando que es el «uso de las TIC en los órganos de la Administración para mejorar la información y los servicios ofrecidos a los ciudadanos, orientar la eficacia y eficiencia de la gestión pública e incrementar sustantivamente la transparencia del sector público y la participación de los ciudadanos» (CLAD 2007, 7).

Resulta oportuno, en la actualidad cubana, analizar un conjunto de criterios que propicien el establecimiento del gobierno electrónico, a partir de un instrumento (hoja de ruta) que instituya para los distintos actores involucrados el ámbito de planificación, ejecución y mejoras del gobierno electrónico; lo que constituye el objetivo de esta investigación. En tal sentido, se analizan los argumentos establecidas en los documentos del 7. ${ }^{\circ}$ Congreso del Partido Comunista- y en la Agenda 2030 de Desarrollo Sostenible, que sostienen la decisión de avanzar hacia el gobierno electrónico. A continuación se esbozan sus características esenciales, el punto de partida actual en el contexto local cubano y, por último, se describen los pasos que permitirán la implementación de forma coherente del gobierno electrónico.

\section{Gobierno y era digital. Razones para implementar el gobierno electrónico en Cuba}

El panorama mundial contemporáneo se caracteriza por las profundas transformaciones originadas en el desarrollo y difusión de las TIC en la sociedad. Al mismo tiempo, el desarrollo de los medios de comunicación y de internet ha acrecentado la responsabilidad del gobierno ante la ciudadanía, al facilitar más participación. Esta era digital, reta la capacidad de respuesta gubernamental ante las crecientes demandas ciudadanas. El ciudadano pasivo es un concepto del pasado. El gobierno enfrenta ahora nuevas formas de participación ciudadana en la toma de decisiones, y debe tener listos

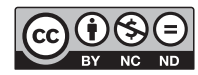


los canales para darles un seguimiento exitoso, en función de elevar la calidad de vida de todos los actores sociales del país.

La relación gobierno-ciudadano es más directa y más exigente en términos de tiempo, dado que las TIC permiten que cada ciudadano participe activa, cercana e inmediatamente en la construcción de las políticas públicas. Los gobiernos deben hallar nuevas formas de operar, de interactuar con la ciudadanía, de organizarse internamente y, con todo ello, ofrecer nuevos conceptos de valor a la sociedad.

En la actualidad cubana, la ampliación del acceso al internet y el incremento de espacios de comunicación ciudadana, reflejan una de las alternativas de información y comunicación más demandadas por los cubanos. Todo esto ha favorecido el intercambio entre diferentes actores sociales que implementan nuevas prácticas de participación sociopolítica, centrada en la satisfacción de sus necesidades y expectativas. En la conceptualización del Modelo Económico y Social Cubano de Desarrollo Socialista, se expone que «la gestión comunicacional promueve la sistematicidad, trasparencia, calidad y oportunidad de la información pública, así como el acceso a ella. Al propio tiempo, el conocimiento, la comunicación e información veraz y oportuna constituyen derechos ciudadanos y bienes públicos ». (Partido Comunista de Cuba 2017, 22).

De igual manera, el Plan Nacional de Desarrollo Económico y Social hasta el 2030 sobrepasa los conceptos clásicos de desarrollo, como el crecimiento económico o el producto interno bruto. Busca mejorar la calidad de vida a través de propuestas que conllevan desafíos técnicos e innovaciones instrumentales, en las que el gobierno electrónico desempeña su rol más importante, como herramienta y potenciador de la mejora en la calidad de vida. Este tipo de gobierno electrónico tiene impactos significativos en los objetivos generales números uno, dos y tres de los ejes estratégicos, que son:

- gobierno socialista, eficaz, eficiente y de integración social;

- desarrollo humano, equidad y justicia social;

- potencial humano, ciencia, tecnología e innovación.

En consecuencia, la explicación ofrecida por las articulistas en relación con cada uno de dichos objetivos, contenidos en el referido Plan Nacional, se expone a continuación:

Objetivo uno del eje estratégico (gobierno socialista, eficaz, eficiente y de integración social): «Consolidar el Estado socialista y democrático, y fortalecer la participación popular decisiva en todas las esferas». El gobierno electrónico, por medio de la participación directa de la ciudadanía y sin que esta deba de forma imperiosa conocer la complejidad natural de la administración pública, con un relacionamiento sencillo y útil, que estimule la participación de todos y todas en las decisiones públicas con una interacción directa tanto del Estado a la ciudadanía, como de ellos a la Administración, reglado en marcos normativos, metodologías y herramientas. (Véase Figura 2). Debe contemplar, además en su modelo, componentes como: la interoperabilidad, que permita el intercambio de información entre los distintos actores de la sociedad; así como un componente que organice la apertura, acceso y gestión de los datos e información para impulsar la coordinación institucional en el comercio.

En el segundo objetivo (desarrollo humano, equidad y justicia social): «Consolidar las conquistas de la Revolución en política social y garantizar el crecimiento progresivo y sostenible del nivel y calidad de vida de la población». El gobierno electrónico tiene influencia directa, que permite al ciudadano acceder a servicios y atención pública de forma oportuna, ágil, transparente y dependiente de un estado eficiente y eficaz. Tiene un impacto destacado en la organización y en la gestión interna de las administraciones públicas. La adaptación y modernización de los aparatos públicos, mediante

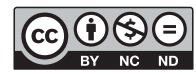


el uso de las TIC con dinámicas transformadoras que promueven el mejoramiento de la calidad en la prestación de servicios, tiene un impacto en la calidad de vida de la población, especialmente a favor de sectores prioritarios.

Por último, en el tercero (potencial humano, ciencia, tecnología e innovación): «Promover el desarrollo, la captación y asimilación de tecnologías de avanzada, y propiciar al mismo tiempo, la soberanía tecnológica». El gobierno electrónico, mediante redes e infraestructura nacional, impulsará procesos transaccionales y participativos para que la ciudadanía acceda en línea a datos, información, trámites y servicios. Las grandes estrategias nacionales buscarán un cambio en la matriz productiva. En relación con este cambio, se debe implementar un gobierno electrónico que en su modelo contemple: el desarrollo del sector de las TIC, que considere al Estado como uno de los motores que, mediante políticas y marcos referenciales, establezca el desarrollo de las soluciones de software; los datos abiertos para que el sector productivo del país tenga información que le permita definir estrategias de negocios, tomar decisiones y desarrollar la innovación, y por último, métodos y herramientas que potencialicen el comercio electrónico. Es imprescindible comprender que «cualquier intento por lograr la superación definitiva del subdesarrollo y la deformación estructural de la economía cubana, que produzca como resultado integral una sociedad próspera, ambientalmente sostenible, inclusiva, justa, crecientemente participativa, y que se inserte de forma competitiva y ventajosa en la economía internacional debe transcender la visión de internet como repositorio documental o servicio de comunicación y asumir su potencial para generar procesos de trasformación, aprendizaje social, vigilancia ciudadana y desburocratización del diálogo entre los individuos y sus gobiernos e instituciones». (Díaz y Sokooh 2013, 64).

En esencia, el Estado debe mejorar su gestión y la calidad en la prestación de sus servicios a la ciudadanía, por lo que el uso de las TIC se convierte en un factor clave. Para su materialización, es indispensable consolidar sinergias interinstitucionales, con el fin de generar formas innovadoras y nuevos espacios de interacción entre el Estado y la sociedad. Estas ideas sitúan a las TIC como un elemento de apoyo para el desarrollo del gobierno, ya que, mediante su uso adecuado, se busca alcanzar mayores niveles de eficiencia y eficacia en el quehacer gubernamental, lo que mejora los procesos y procedimientos del gobierno, aumenta la calidad de los servicios públicos, incorpora más y mejor información en los procesos decisorios y facilita la coordinación entre las diferentes instancias de gobierno.

El gobierno electrónico no es un fin en sí mismo: tiene un carácter instrumental que requiere la revisión, rediseño y optimización de los procesos como paso previo a la introducción de cualquier cambio en la tecnología o en las funciones de producción de las organizaciones públicas. De esta manera, adquiere una doble dimensión: de agente catalizador de los cambios en los procesos y de herramienta tecnológica, como instrumento para mejorar el desempeño de los actos del Estado.

\section{Gobierno electrónico y la Agenda 2030 de Desarrollo Sostenible}

En septiembre de 2015, el Estado cubano participó en la adopción de la Agenda 2030 de Desarrollo Sostenible, cuyo compromiso es lograr un mundo más próspero, en paz, justo e incluyente durante los próximos quince años. A los efectos de su cumplimiento, Cuba apuesta al uso de las TIC para establecer nuevos horizontes en la prestación de servicios públicos, así como nuevas estrategias para alcanzarlos.

Al respecto, el objetivo 16 de Desarrollo Sostenible propuesto, se centra en: la promoción de sociedades pacíficas e inclusivas para el desarrollo sostenible, la provisión de acceso a la justicia para todos y la construcción de instituciones responsables y eficaces a todos los niveles. Una de las vías que contribuirá a su cumplimiento es el gobierno electrónico que busca, fundamentalmente, los aspectos siguientes:

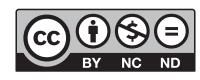


- El uso y despliegue de las TIC al seguir determinadas pautas, normas, experiencias y buenas prácticas; que faciliten el acceso público a la información y la protección de las libertades fundamentales, de conformidad con las leyes nacionales y los acuerdos internacionales.

- Proveer la oportunidad de plantear una nueva forma de hacer gobierno al crear, en todos los niveles, instituciones eficaces y transparentes que rindan cuentas, y garanticen la adopción en de decisiones inclusivas, participativas y representativas que respondan a las necesidades de la ciudadanía. Es significativo el papel del gobierno electrónico con la intervención ciudadana en la creación de medios electrónicos para la asistencia en línea, construcción de políticas públicas; así como los medios electrónicos de publicación de los resultados, calendarios interactivos con las distintas actividades establecidas para la participación ciudadana, y medios electrónicos para la retroalimentación de la prestación de servicios públicos y su mejora.

- Orquestar y gestionar, de forma coherente: personas, tecnologías, normas, servicios, sistemas y procesos para propiciar el acceso a sitios web de ministerios. Así como la conexión de estos con una ventanilla única virtual a nivel nacional, que permita concentrar los servicios electrónicos, la información respecto a estos servicios con un modelo de autorización para el uso de los mismos, la estructuración de la seguridad en los servicios electrónicos, la organización del uso de certificados electrónicos, el desarrollo de la firma electrónica y el pago electrónico, así como la implementación de mecanismos para permitir el acceso de personas con discapacidades y los servicios de publicación de oportunidades de trabajo.

\section{Arquitectura del marco regulatorio del gobierno electrónico}

La organización del gobierno electrónico se fundamentará en una arquitectura del marco regulatorio, que será definida a nivel nacional; en la cual, las instituciones de la Administración Pública Central, diseñarán, planificarán, elaborarán y controlarán las diferentes leyes, decretos, resoluciones, dictámenes, normas e instructivos para el establecimiento del gobierno electrónico cubano. La arquitectura permitirá organizar y estandarizar la implementación y mejora continua de los servicios electrónicos, así como mejoras en general a la gestión pública. El análisis de este marco regulatorio en varios países de América Latina permitió identificar un conjunto de documentos representativos, que integran el marco regulatorio en estos países y que, sin dudas, constituyen referencias orientadoras para Cuba (Figura 1).

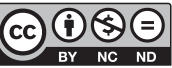




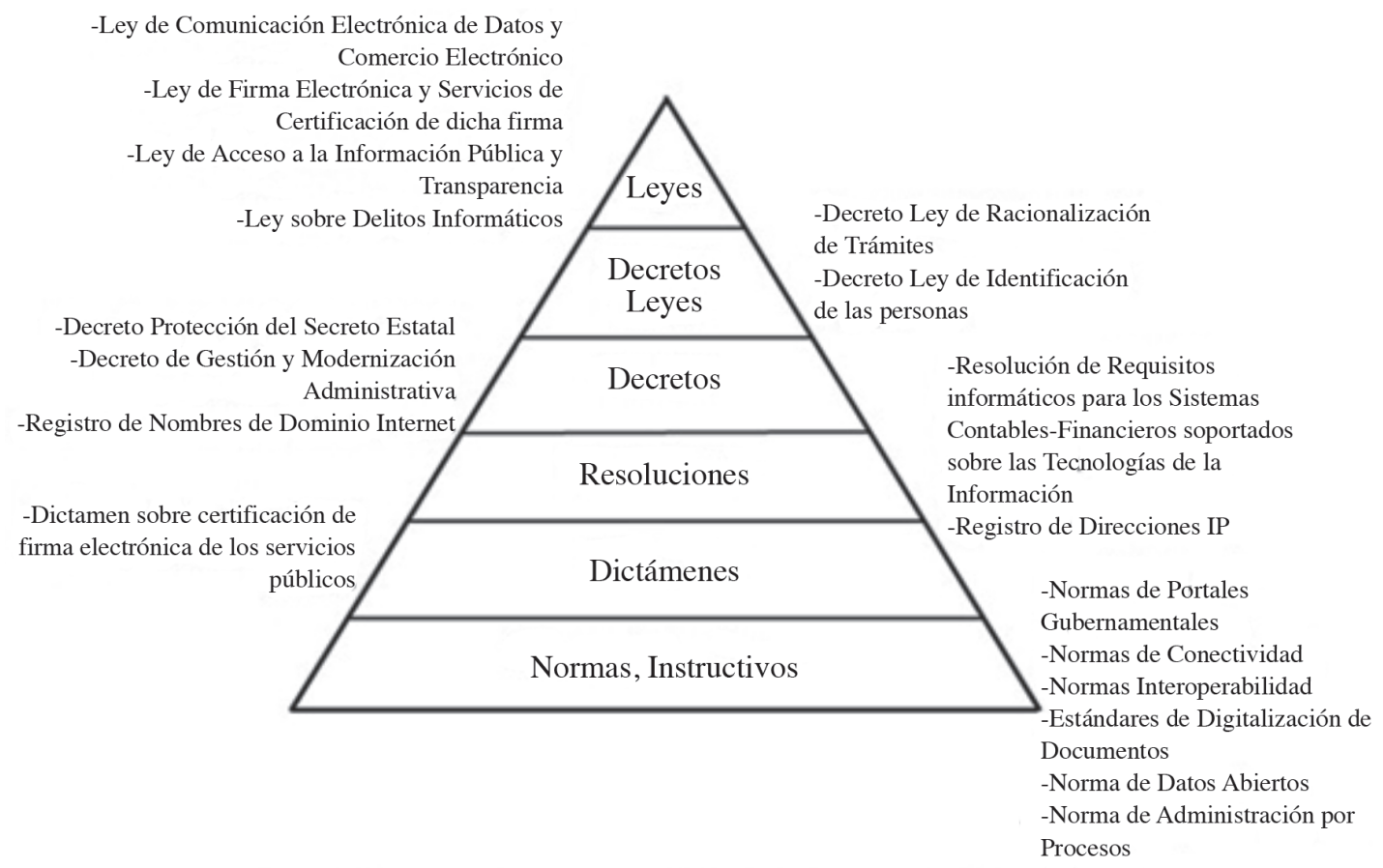

Fuente: Elaboración propia.

El comportamiento general, por países, de la dinámica representada en la figura 1, se muestra a continuación:

- Ley de Comunicación Electrónica de Datos y Comercio Electrónico: Bolivia, Chile, Ecuador, Venezuela, R. Dominicana, Nicaragua, Uruguay.

- Ley de Firma Electrónica y Servicios de Certificación de dicha firma: Bolivia, Chile, Ecuador, Venezuela, R. Dominicana, Nicaragua, Uruguay.

- Ley de Acceso a la Información Pública y Transparencia: Chile, Ecuador, R. Dominicana, Venezuela, Bolivia, Nicaragua, Uruguay, Brasil, Paraguay, Panamá, Perú, México, Colombia, El Salvador, Costa Rica.

- Ley sobre Delitos informáticos: Chile, Venezuela, Uruguay.

- Decreto Ley de Racionalización de Trámites: Venezuela, Nicaragua, Chile.

- Decreto Ley de Identificación de las Personas: Venezuela.

- Decreto de Protección del Secreto Estatal: Cuba, Chile.

- Decreto de Gestión y Modernización Administrativa: Bolivia, Perú, Ecuador.

- Registro de Nombres de Dominio Internet: Bolivia.

- Resolución de Requisitos Informáticos para los Sistemas Contables - Financieros soportados sobre las TIC: Cuba.

- Registro de Direcciones IP: Cuba.

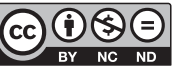


- Dictamen sobre Certificación de Firma Electrónica de los Servicios Públicos: Chile, Brasil, Venezuela, Uruguay, Ecuador.

- Norma de Portales Gubernamentales: R. Dominicana, Ecuador, Chile.

- Norma de Conectividad: R. Dominicana, Ecuador, Chile.

- Norma de Interoperabilidad: R. Dominicana, Chile, Ecuador.

- Estándares de Digitalización de Documentos: Ecuador, Chile, Uruguay.

- Norma de Datos Abiertos: Argentina, Brasil, Colombia, Costa Rica, México, Perú y Uruguay (en la actualidad, estos siete países han consolidado sus políticas nacionales de datos abiertos que sientan las bases legales y técnicas para la apertura de datos abiertos). De acuerdo con (BID 2018, 13- 14).

- Norma de Administración por Procesos: Ecuador y Perú.

El análisis de la arquitectura del marco regulatorio del gobierno electrónico, ha permitido valorar la conveniencia de esta infraestructura legal para la sucesiva mejora de la eficacia y calidad de la gestión pública, y su consecuente modernización, a tono con las exigencias de la ciudadanía en el siglo XXI y el desarrollo de la cuarta revolución industrial.

\section{Relaciones del Gobierno Electrónico}

El gobierno electrónico organiza las TIC de la manera más conveniente, para mejorar la forma de relacionarse por parte de los cuatro actores principales (gobierno, ciudadanía, sector productivo y servidores públicos); y eliminar de esta forma las barreras de comunicación, así como fortalecer las relaciones y alianzas con los actores de una sociedad. Las interacciones que se establecen en el gobierno electrónico se describen a continuación:

\section{Interacción gobierno para el ciudadano (IGC). -}

Son iniciativas encaminadas a brindar servicios públicos e información a la ciudadanía mediante las TIC. La idea es que, las ciudadanas y ciudadanos, puedan interactuar con el gobierno por cualquier medio que le provea acceso, en cualquier lugar y a toda hora. Los beneficios que busca este modelo son principalmente ahorros de tiempo y dinero, ya que se deja de lado la necesidad de desplazarse a las oficinas públicas, donde generalmente debe esperar un tiempo considerable para recibir un servicio público o recibir información.

\section{Interacción gobierno para el gobierno (IGG). -}

Son las interacciones complementarias e interdependientes entre las distintas instituciones del sector público para fomentar eficiencia en la gestión. Los principales beneficios son: evita la duplicidad de procedimientos y agilita los trámites entre instituciones públicas. Asimismo, implica el relacionamiento con otros gobiernos para desarrollar estrategias comunes que apalanquen la madurez del gobierno electrónico.

\section{Interacción gobierno para el sector productivo (IGP). -}

La finalidad es facilitar a este último el acceso a incentivos, productos y servicios públicos. El principal beneficio es la reducción de costos de producción, incremento de la competitividad y la

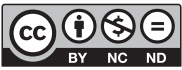


consolidación de un entorno más seguro, ágil y eficiente para la actividad productiva. Adicionalmente, el gobierno se beneficia de una mejor gestión tributaria.

\section{Interacción gobierno para los servidores públicos (IGS). -}

Es la gestión que desarrolla un gobierno para brindar servicios de desarrollo profesional y atención a las demandas de su talento humano. En ese sentido, apalanca la gestión del talento humano desde el proceso de reclutamiento hasta su desvinculación. El principal beneficio de este modelo de relación, es que se cuenta con herramientas y mecanismos que permiten fortalecer las competencias de los servidores públicos.

En la Figura 2 se muestran las distintas interacciones de los actores que intervienen en el gobierno electrónico.

Figura 2

Interacciones de los cuatro actores del gobierno electrónico

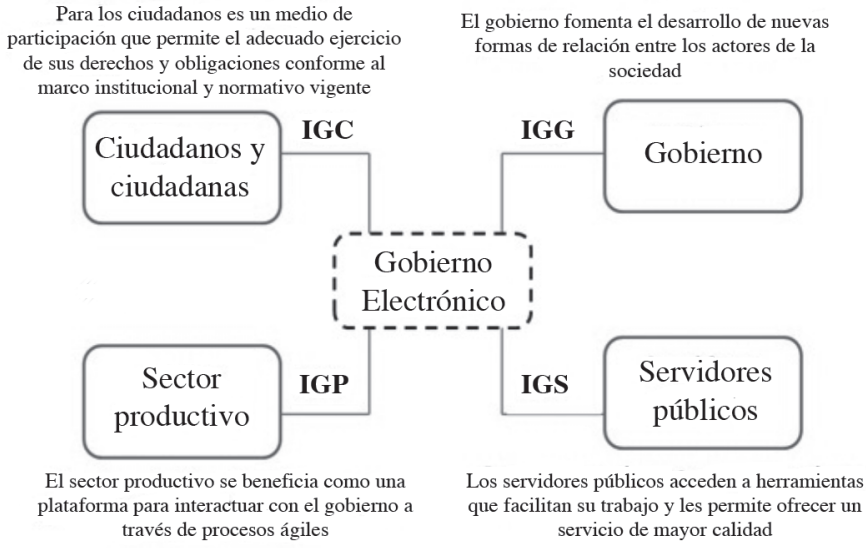

Fuente: Elaboración propia.

La ciudadanía podrá relacionarse electrónicamente con los gobiernos y las administraciones públicas. Asimismo, entre otras actividades, se encuentran las siguientes:

- Dirigir, por vía electrónica, todo tipo de escritos, recursos, reclamaciones y quejas a los Gobiernos y las Administraciones Públicas. Por lo que estos quedan obligados igualmente a responder o resolver como si dichos escritos, reclamaciones y quejas, se hubieran realizado por medios tradicionales.

- Realizar, por medios electrónicos, todo tipo de pagos, así como presentar y liquidar impuestos, y cualquier otra clase de obligaciones. 
- Acceder, por medios electrónicos, a la información administrativa general; con igual grado de fiabilidad que la que es objeto de anuncio en diarios o boletines oficiales, o la que se publica en anuncios oficiales por cualquier medio.

- Acceder a los expedientes electrónicamente, quienes estén interesados, para conocer el estado en que se encuentra la tramitación de los mismos.

- Utilizar y presentar, ante el gobierno o las administraciones públicas, las resoluciones en soporte electrónico; así como los documentos oficiales en iguales condiciones que si fueran documentos en papel, para poder remitirlas por medios electrónicos a la administración de que se trate.

\section{Aspectos vitales para la implementación sostenible del gobierno electrónico}

- Transformaciones de las Administraciones Públicas. -

Se deberán acometer las transformaciones organizativas que se consideren necesarias para establecer programas de adaptación de los empleados públicos a los nuevos sistemas de gobierno electrónico. De igual manera, promover la profesionalización de los mismos, a fin de conservar y desarrollar las nuevas competencias para garantizar de esta manera la sostenibilidad en el tiempo de la ejecución de los procesos electrónicos, y así adaptar las reglas de procedimiento a las posibilidades que ofrecen las comunicaciones electrónicas.

- Integración de procesos y servicios. -

Primero, las organizaciones deberán levantar la información de los procesos en su situación actual, con la finalidad de identificar todas las actividades, recursos, tecnologías, controles, reglas de negocio y datos estadísticos que se ejecutan en los procesos; lo cual servirá para su posterior diagramación y análisis. Se debe realizar un análisis sobre la problemática de los procesos, para poder identificar sus causas. En el caso de que las acciones de mejora incluyan automatización, se deben definir, previamente: los requerimientos funcionales, las especificaciones técnicas y los resultados de la ejecución de los procesos bajo diferentes escenarios. Se debe orientar el gobierno electrónico a ofrecer servicios públicos integrados, así como propender a una mayor integración de sus procesos internos. Se recomienda el desarrollo de portales únicos que agrupen diversos servicios electrónicos.

- Despliegue de las infraestructuras. -

Para la universalización del gobierno electrónico, es necesario el despliegue de las infraestructuras que sean capaces de dar soporte a las velocidades de transmisión; para que el acceso al gobierno electrónico constituya una posibilidad real, en la medida en que los tiempos de acceso a los servicios no impliquen retardos. Es imprescindible el desarrollo de la banda ancha, mediante tecnologías que permitan un tiempo de respuesta adecuado para el usuario.

- La interoperabilidad de servicios. -

Interconexiones entre las organizaciones, y sistemas dispares y diversos para interactuar con objetivos consensuados y comunes, con la finalidad de obtener beneficios mutuos. (Criado, Gascó y

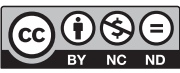


Jiménez 2010, 5). La interoperabilidad de servicios es una necesidad para maximizar la cooperación entre las organizaciones. En la Figura 3 se describen las tres dimensiones que generalmente se referencian en la literatura consultada.

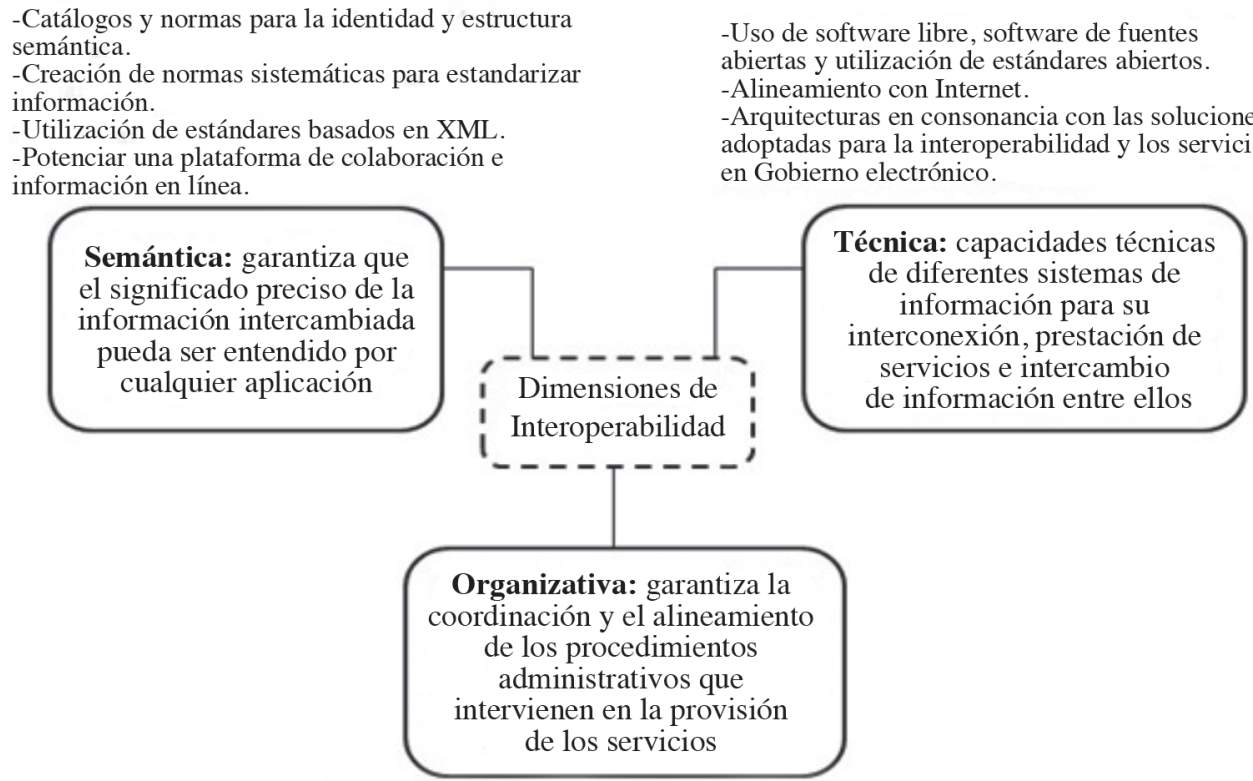

-Modelización de los procesos administrativos para lograr una visualización de los flujos de actividades que los componen.

-Diagramación de los flujos internos.

-Nuevo liderazgo para aunar los conocimientos y capacidades gerenciales

tradicionales de la gestión pública directa (estrategia, personal, organización, etc.) junto con los de gestión de redes.

-Normativa sobre identidad digital, privacidad de los ciudadanos y protección de los datos personales y seguridad en la interoperabilidad.

Fuente: Elaboración propia.

- Inclusión digital e infoalfabetización. -

El estado debe establecer políticas, estrategias y programas de inclusión digital; para combatir la infoexclusión, reducir la brecha digital y eliminar las barreras existentes para el acceso a los servicios electrónicos. Se debe ampliar el acceso de los sectores populares a las TIC y promover, de forma general, una cultura ciudadana sobre el gobierno electrónico. La transformación de la cultura social en cuanto al gobierno electrónico, con un proceso de formación ciudadana e infoalfabetización que estimule el acceso, participación y utilización del mismo, es un elemento fundamental de ese proceso de preparación de la ciudadanía.

- Usabilidad de sistemas y programas. -

La usabilidad expresa que los programas tienen que ser disponibles, accesibles y manejables intuitivamente por el ciudadano. Paralelamente, en lo que hace a la producción de contenidos a través de sitios Web u otros medios electrónicos, deberán incorporarse mecánicas de personalización

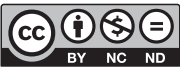


de sectores específicos para seleccionar los lenguajes utilizados, e identificar permanentemente los perfiles de usuarios y sus correspondientes necesidades de información y servicios, al poner especial atención en la inclusión de personas con discapacidades. También, se deberá utilizar un lenguaje simple, directo y evitar el uso de siglas.

\section{Punto de partida actual en el contexto local cubano}

El gobierno cubano ha identificado la necesidad de introducir las TIC en las actividades de la administración pública, para contribuir a la eficacia y eficiencia de los servicios prestados a todos los ciudadanos y ciudadanas. Una derivación de esta política es el "Programa Rector para la informatización de la sociedad cubana", que propicia el uso masivo de las TIC a escala nacional, al considerar los objetivos estratégicos trazados por el gobierno cubano. Un conjunto de inversiones destinadas a modernizar la infraestructura ha propiciado el desarrollo de la telefonía móvil e internet, así como el subsidio de sectores, tales como la educación, la salud y la cultura, entre otros. La conectividad en espacios públicos (bibliotecas, parques y la distribución de contenidos por medio de los teléfonos móviles) constituyen ejemplos de la voluntad política de los actores decisores para fomentar una cultura digital en beneficio de satisfacer las demandas sociales.

Análisis recientes del grupo de investigación de la administración pública de la Universidad de Holguín, con 30 expertos, sobre las debilidades que coexisten en el desarrollo del gobierno electrónico, permitió constatar que los factores que lo obstaculizan son los siguientes:

1. Barreras culturales y normativas.

2. La hostilidad de algunas dependencias del gobierno americano y otros actores aislados, que emplean las TIC para subvertir e influenciar, en los ciudadanos y ciudadanas del país, el cambio del sistema político actual.

3. Fragmentación administrativa que facilita la convivencia de varios sistemas informacionales en la misma administración pública; y, casi siempre, sin que exista interoperabilidad entre los modelos de tecnologías de la información instalados.

4. El uso de las TIC se transforma en un fin en sí mismo, y no es considerado como un medio para alcanzar objetivos formulados en la organización.

5. Procesos poco flexibles y complejos, enfocados a los requisitos de los decisores internos, con varios puntos de control que no añaden valor a los requerimientos de los beneficiarios a los servicios públicos.

6. Infraestructura que limita la dinámica de los trámites en línea, debido a la lentitud y limitación de la conexión.

7. Existencia de brecha tecnológica entre lo urbano y lo rural.

De igual manera, se establecieron las fortalezas siguientes:

1. Voluntad política de los decisores del gobierno cubano, para desarrollar la infraestructura y el marco regulatorio necesario para garantizar la construcción sostenible de la estrategia de informatización de la sociedad cubana.

2. Apertura de un creciente número de salas públicas de navegación, con alcance nacional y subnacional.

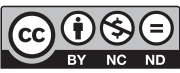


3. Existencia de un capital intelectual, con altas competencias técnicas en el sector de la informática y telecomunicaciones, para desarrollar el gobierno electrónico en Cuba.

4. Implementación gradual de numerosos proyectos para estructurar una plataforma informática nacional que asegure la realización y distribución de contenidos en el país, gestionados por organizaciones cubanas y que reflejen los valores patrios e identidad nacional.

5. Creación de nuevas capacidades de acceso a nuevas tecnologías y el despliegue de redes institucionales, como las del sector de la salud (infomed), sostenidas en servidores de altas prestaciones para satisfacer las demandas crecientes del polo científico de la salud.

6. Introducción de la televisión digital en el país, que moderniza la infraestructura televisiva.

7. Las demandas crecientes de los ciudadanos y ciudadanas, mediante los diferentes mecanismos de participación instituidos por el gobierno, de recibir servicios eficientes y eficaces que les permitan obtener mayor valor público, y la disminución de los tiempos de prestaciones de los servicios públicos generados en el país.

En general, la balanza se inclina hacia el trabajo sistemático para hacer avanzar la implementación de acciones que conlleven a la administración digital o gobierno electrónico en Cuba. En recientes publicaciones (Real Castro, Medina López, Rojas Ríos, Lazo Brito, Paredes Álvarez y Vento Tielves 2018; Rodríguez Blanco, Ricardo Soto y Sariol Guerra 2018; Armas Urquiza y Armas Suárez 2017), se recogen experiencias que van dirigidas en este sentido, tales como: "el Portal del Ciudadano de Pinar del Río, la plataforma WEB para el Gobierno Provincial de Granma y los resultados presentados de varias unidades de observación, establecidos en los Consejos de la Admistración Municipal de la capital cubana”.

Estas experiencias cubanas se dirigen, fundamentalmente, a publicar el quehacer del gobierno; con la finalidad de que la población conozca lo que este hace en la localidad, a favor del bienestar de la ciudadanía. Igualmente, promueven la participación ciudadana mediante comentarios, foros y formularios para el envío de fotos de la ciudad, y para contactar con el gobierno y expresar opiniones, sugerencias o quejas. Se trabaja en aplicaciones para facilitar trámites demandados en las Asambleas del Poder Popular, y hasta la fecha esta iniciativa ha tenido una atractiva acogida local.

\section{Hoja de ruta para el establecimiento del gobierno electrónico en Cuba}

La organización encargada de la gestión del gobierno electrónico, en cada provincia y municipio, definirá cómo organizar, de acuerdo con las peculiaridades de su contexto, los pasos para la implementación del gobierno electrónico que se sugieren a continuación:

\section{PASO 1: Preparación para el cambio hacia el gobierno electrónico}

Objetivo: minimizar la resistencia al cambio en los actores involucrados para facilitar el camino hacia el establecimiento del gobierno electrónico.

Participantes: Actores involucrados del gobierno y expertos en la temática.

\section{Actividades:}

- La necesidad de establecer el gobierno electrónico, la cual consiste en la impartición de seminarios, conversatorios y debates, que van dirigidos a los actores involucrados de las organizaciones que participarán en el proyecto, para de esta forma adquirir el conocimiento y la comprensión sobre la temática.

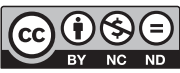


- El cambio hacia el estado deseado para originar una reflexión en los funcionarios públicos, con vistas al reconocimiento y aprehensión de lo nuevo, que favorezca la minimización de la resistencia natural.

- El inicio de la instauración del gobierno electrónico, en el que debe nombrarse al equipo líder del proyecto (se recomienda ser presidido por un miembro de los funcionarios como su representante), designar responsabilidades, atribuciones de sus miembros y elaboración del plan de trabajo.

\section{Resultados:}

- Funcionarios públicos sensibilizados y comprometidos.

- Equipo de proyecto organizado.

\section{PASO 2: Realizar diagnóstico detallado de la situación actual}

Objetivo: realizar una revisión global de los recursos con los cuales se desarrollarán las distintas soluciones del gobierno electrónico; o sea: el marco regulatorio vigente (instrumentos legales y jurídicos que permiten la construcción de soluciones del gobierno electrónico), los servicios y procesos (actividades institucionales que generan valor añadido a la ciudadanía) ofertados por las organizaciones públicas, las TIC (conjunto de recursos tecnológicos usados en la captura, el procesamiento, el almacenamiento y la transmisión de información); que han sido desplegadas en el país y las personas (talento humano que genera y articula de manera estratégica las normativas, procesos y tecnologías de la información y comunicaciones), o actores que tendrán participación directa en su desarrollo.

Participantes: equipo de proyecto

Actividades:

- El marco regulatorio vigente será identificado exhaustivamente.

- Los servicios y procesos ofertados a la ciudadanía serán analizados en correspondencia con las normativas aplicables y las demandas de la población en cuestión.

- Las TIC disponibles serán analizadas, según las exigencias del gobierno electrónico.

- El gobierno electrónico requerirá determinadas competencias de las personas que intervendrán en su ejecución.

Resultados:

- Inventario existente del marco regulatorio, los servicios y procesos, las TIC y las personas.

- Definición del punto de partida para el gobierno electrónico en Cuba.

\section{PASO 3: Determinar los objetivos, estrategias e indicadores del gobierno electrónico}

Objetivo: formular los objetivos del gobierno cercano, eficiente y eficaz, las estrategias e indicadores del gobierno electrónico.

Participantes: decisores del gobierno y equipo de proyecto

Actividades:

- El número de los servicios públicos a los cuales el ciudadano puede acceder en línea, a través de cualquier dispositivo, en cualquier lugar y a cualquier hora será determinado por los participantes del proyecto.

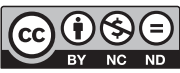


- La información pública útil en formatos abiertos y reutilizables se delimitará para que la ciudadanía colabore y participe activamente en el proceso de toma de decisiones del gobierno.

- El grado de informatización de los servicios y procesos en función de las demandas y expectativas de la ciudadanía, así como la reducción del tiempo de prestación del servicio, se definirá por dichos decisores.

- La formulación de los indicadores, tales como: el número de servicios en línea, índice de percepción de calidad de los servicios públicos, porcentaje de instituciones de la administración pública central, que cuentan con sitios web accesibles, número de cursos virtuales, número de instituciones con datos abiertos y de aplicaciones en el portal de software público, porcentaje de procesos claves automatizados, entre otros indicadores.

Resultados:

- Planificación del gobierno cercano a la ciudadanía, eficiente y eficaz.

\section{PASO 4: Determinar los recursos para cumplir los objetivos y estrategias formuladas}

Objetivo: identificar los recursos requeridos del gobierno electrónico planificado (marco regulatorio, los servicios y procesos, las TIC y las personas).

Participantes: equipo de proyecto

Actividades:

- Las leyes, decretos, normas e instructivos que serán necesarios para documentar y sustentar el funcionamiento eficiente del gobierno electrónico planificado, deberá ser determinado consecuentemente.

- Cada sector definirá los servicios electrónicos que van a proveer por parte de las instituciones competentes. Es recomendable considerar como prioridad los servicios electrónicos que están enmarcados en: educación, salud, justicia y seguridad ciudadana.

- Los recursos tecnológicos necesarios para la captura, el procesamiento, el almacenamiento y la transmisión de información en el gobierno electrónico planificado serán definidos.

- Las necesidades de personas que generarán y articularán de manera estratégica las normativas, procesos y tecnologías de la información y comunicaciones serán establecidas.

Resultados:

- Marco regulatorio: leyes, decretos, normas e instructivos requeridos (ley de gobierno electrónico, ley de transparencia y acceso a la información pública, decreto de software libre y estándares abiertos, decreto de gobierno electrónico y simplificación de trámites, decreto de firma electrónica, norma de datos abiertos y de utilización de nube de gobierno, normativa de interoperabilidad y de generación de contenidos de capacitación, entre otros documentos).

- Servicios y procesos: portafolio y catálogo de servicios, carta de servicios, inventario de procesos que generan valor público en los sectores priorizados.

- TIC: plataforma de interoperabilidad y de autentificación, red de alta velocidad, centro de servicios compartidos, anillo interministerial (interfaz de datos gubernamental, distribuida por fibra óptica para la transmisión de información ministerial en una red interconectada de ministerios, secretarías y otras instituciones en línea) y anillo institucional.

- Personas competentes y flexibles que propicien el desarrollo sostenible del gobierno electrónico.

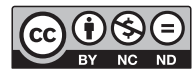




\section{PASO 5: Desglosar el presupuesto asociado a los recursos identificados}

Objetivo: disponer de los recursos financieros para la ejecución real del gobierno electrónico.

Participantes: equipo de proyecto

Actividades:

- El presupuesto asociado a la elaboración del marco regulatorio necesario será determinado, así como la construcción de los servicios y procesos informatizados e integrados. A la par, las TIC que soportarán la ejecución de los servicios y procesos que integrarán el gobierno electrónico, y la formación y entrenamiento de las personas que participarán en el mismo.

Resultados:

- Aprobación de los presupuestos del gobierno electrónico.

\section{PASO 6: Diseñar la realización de una prueba piloto del gobierno electrónico}

Objetivo: desarrollar un periodo de transición para consolidar y perfeccionar el gobierno electrónico en un municipio del país, para su posterior generalización.

Participantes: equipo de proyecto

Actividades:

- El equipo de proyecto y los expertos elaborarán un plan para desplegar el gobierno electrónico, como parte del inicio y preparación de la prueba.

- Se dispondrán los recursos necesarios y se realizarán los cambios que se deriven de la implantación de los nuevos procedimientos establecidos o de las recomendaciones del personal, así como su aprobación y comunicación a todas las partes interesadas relevantes, con vistas a la implantación del electrónico.

- Se realizarán los ajustes necesarios para cumplir los objetivos trazados por el equipo y se controlarán los cambios para formalizarlos y comunicarlos, como función de este proceso de la implantación.

- Para la generalización en todo el país del gobierno electrónico, se analizarán los resultados alcanzados en la prueba piloto, y se tendrá en cuenta la opinión de todas las personas que participaron en la misma. Del mismo modo, se determinarán los puntos de control que permitirán asegurar que el gobierno electrónico se pueda desplegar según lo planificado, para detectar a tiempo las posibles desviaciones, y analizar las causas para su solución.

Resultados:

- Contextualización y consolidación de los procedimientos establecidos en el gobierno electrónico.

\section{PASO 7: Evaluar los objetivos planificados del gobierno cercano, eficiente y eficaz}

Objetivo: seguir, medir, analizar y evaluar en el país, los objetivos formulados a través de los indicadores planificados.

Participantes: equipo de proyecto

Actividades:

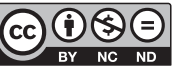


- El número de los servicios públicos a los cuales el ciudadano puede acceder en línea, por cualquier dispositivo, en cualquier lugar y a cualquier hora, así como del porcentaje de procesos claves automatizados serán evaluados convenientemente.

- El índice de percepción de calidad de los servicios públicos será evaluado para constatar el cumplimiento del valor planificado.

- Se determinará el porcentaje de instituciones de la administración pública central, institucional y dependiente de la función ejecutiva que cuentan con sitios web accesibles.

- Se valorará el número de cursos virtuales existentes, de instituciones con datos abiertos, de aplicaciones en el portal de software público, de servicios en línea que cuentan con datos abiertos, del porcentaje de instituciones que usan los sistemas gubernamentales y de instituciones oficiales que publican servicios web para consumo de otras entidades con estándares de interoperabilidad.

\section{Resultados:}

- Evidencias del estado del gobierno electrónico.

En la formulación de los indicadores será necesario considerar los aspectos siguientes: nombre del indicador, descripción del indicador, línea base, unidad de medida, fuente, método de cálculo, frecuencia de actualización y la meta planificada. Por ejemplo, para medir el número de servicios en línea, ofertados en el portal único, el indicador se formulará de la manera siguiente:

Nombre del indicador: número de servicios en línea ofertados a través del portal único

Descripción del indicador: este indicador muestra la cantidad de servicios que han desarrollado las instituciones oficiales y que son publicados dentro del portal único gubernamental

Línea base: 0

Unidad de medida: número

Fuente: portal único gubernamental

Método de cálculo: conteo de servicios publicados en un portal único

Frecuencia de actualización: semestral

Meta (2018): 105 servicios en línea

\section{PASO 8: Revisión y priorización de mejoras del gobierno electrónico}

Objetivo: revisar y determinar las mejoras sistemáticas que se priorizarán, a partir de considerar su costo, el tiempo de ejecución y el impacto de estas en la calidad de vida de la ciudadanía.

Participantes: alta dirección de la administración pública y equipo de proyecto Actividades:

- La alta dirección de la administración pública y el equipo de proyecto analizarán las diferentes alternativas (mejora continua, reingeniería de procesos, innovación, otras alternativas) que existan para la realización de mejoras del gobierno electrónico implementado.

- Las mejoras que serán priorizadas en función de las variables de costo, tiempo de realización e impacto en la calidad de vida de la ciudadanía, serán establecidas para un mejor funcionamiento del gobierno electrónico.

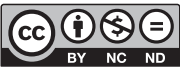


- Los recursos deberán planificarse y suministrarse de forma oportuna. El equipo documentará las mejoras ejecutadas para poder evaluarlas en el tiempo; e institucionalizarlas, para propiciar que los actores involucrados conozcan de los nuevos procedimientos implementados.

Resultados:

- Plan de formación y entrenamiento de actores involucrados en los nuevos procedimientos institucionalizados y evidencias de su ejecución.

- Mejoras (recursos requeridos) del gobierno electrónico y evidencias de su ejecución.

\section{Conclusiones}

La finalidad esencial del gobierno electrónico es incrementar la calidad de los servicios y productos públicos que la administración pública suministra a la ciudadanía. Es un proceso complejo y requiere de tiempo, y no se trata solo de que la ciudadanía pueda estar informada del quehacer de la administración pública y de pagar los impuestos por internet sino que, además puedan tener una discusión pública acerca de cómo se gastan esos impuestos. El desafío es ir más allá de los modelos unidireccionales de servicio y políticas públicas: se trata de la inclusión del ciudadano dentro de la toma de decisiones durante todo el ciclo de vida de la gestión pública.

Se aprecia que, se deberán generar por las instituciones pertinentes, los elementos del marco regulatorio que permitan la construcción de soluciones del gobierno electrónico, para garantizar la operatividad, calidad, sostenibilidad y funcionalidad de las mismas. De igual manera, estos instrumentos también regularán y guiarán el accionar de los distintos actores involucrados.

Es necesario realizar el levantamiento, estandarización y optimización de procesos gubernamentales transversales, antes de realizar la automatización de los mismos; incluso antes de la automatización, se debe asegurar que las normativas y regulaciones estén aprobadas. Esto evitará duplicación de esfuerzos y desperdicio de fondos públicos.

El Modelo Económico y Social Cubano de Desarrollo Socialista, los Lineamientos para el periodo 2016-2021, el Plan Nacional de Desarrollo Económico y Social hasta el 2030, establecen directrices para el bienestar de toda la ciudadanía del país, los que deberán materializarse de acuerdo con una gestión pública moderna, alineada a la era digital, eficaz y eficiente.

El despliegue del gobierno electrónico es una vía probada en varios países de América Latina y el Caribe, que ha mejorado las interacciones: del gobierno para la ciudadanía, gobierno para el gobierno, gobierno para el sector productivo y gobierno para los servidores públicos; lo que permite consolidar un Estado cercano, eficiente y eficaz. La hoja de ruta recomendada contribuirá a la organización y mejora de este proceso. La preparación para el cambio y la evaluación de indicadores debidamente formalizados, es un paso determinante para la consolidación de la gobernabilidad democrática cubana.

\section{Referencias}

Armas Urquiza, Roberto y Alejandro Armas Suárez. 2017. «La informatización de la sociedad. Conceptos y realidades». Administración Pública.

BID. 2018. Los datos abiertos en América Latina y el Caribe. http:// www.iadb.org/

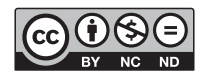


CLAD. 2007. «Carta Iberoamericana de Gobierno Electrónico». Documento aprobado en IX Conferencia Iberoamericana de Ministros de Administración Pública y Reforma del Estado, Chile.

Criado, Ignacio, Mila Gascó y Carlos Jiménez. 2010. «Bases para una Estrategia Iberoamericana de Interoperabilidad». Documento para la consideración de la XII Conferencia Iberoamericana de Ministros de Administración Pública y Reforma del Estado Buenos Aires, Argentina. http:// www.latinobarometro.org/

Díaz Rodríguez, Elaine y Firuzeh Sokooh Valle. 2013. «Internet y las TIC en Cuba: notas para un debate sobre políticas públicas». Tema, 74 (2). Ciudad de La Habana, Cuba. Impreso en Canadá.

Partido Comunista de Cuba 2017. «Modelo económico y social cubano de desarrollo socialista». 7mo Congreso del Partido Comunista de Cuba, 2-13. Gaceta oficial de la República de Cuba.

Real Castro, Elena Julia et al. 2018. «Portal del ciudadano: una herramienta de gobierno electrónico en Pinar del Río». 17 Convención y Feria Internacional Informática 2018. La Habana, Cuba.

Rodríguez Blanco, Yanais Yisel, Reinerio Ricardo Soto y Yisel Sariol Guerra 2018. «Sistema de gobierno electrónico de la República de Cuba. Experiencia en la provincia de Granma». 17 Convención y Feria Internacional Informática 2018. La Habana, Cuba. 